\title{
Thomas Groß \\ Grenzen der Pluralisierung der Judikative \\ Das Gebot struktureller Unparteilichkeit ehrenamtlicher Richter
}

Mit der Diskussion über Justizreformen im Bereich der Gerichtsverfassung geraten auch die ebrenamulichen Richter unter Rechtfertigungszwang. Dabci sollee allerdings nicht nur das modische Ziel der "Verschlankung" berücksichtigt werden, sondem auch die Funktion des Laienrichtertums kritisch überprüft werden. Der Beitrag untersucht diese Frage unter dem speziellen Gesichispunkt der verfassungs- und europarechtlichen Garantie der Unparteilichleit des Richters.

Die Red.

\section{Problemsiellung}

\section{Der Fall Langborger}

Verstößc der Einsatz ehrenamtlicher Richter, diê auf Vorschlag von Arbeitnehmerbzw. Arbeitgebervereinigungen bestellt werden, in den Arbeitsgerichten gegen Art. 6 Abs. I EMRK, der jedermann das Recht gewährleistet, daß seine Sache von einem unabhängigen und unparteiischen, auf Geserz beruhenden Geriche gehört wird? Diese auf den ersten Blick überraschende Fragestellung wird durch ein Urteil des Europäischen Gerichtshofes für Menschenrechce' aufgeworfen, das bisher auf deutsch nicht veröffentlicht worden ist, dessen Ratio aber weit über den entschiedenen Fall hinausreicht.

Der schwedische Beschwerdeführer harre Klage erhoben, weil er sich von einer zuvor vertraglich vereinbarten Klausel lösen wollte, nach der aufgrund einer spezieilen Regelung des schwedischen Rechts die Miechöhe und die Vertragsbedingungen durch einen Kollektivvertrag der nationalen Vereinigungen der Hauseigentümer bzw. der Mieter festgelegt werden. Für Strcirigkeiten aus solchen Mietverträgen richtete das schwedische Recht eine besondere Gerichtsbarkeit ein. Der Spruchkörper der zweiten Instanz bestand aus vier Mitgliedern, zwei Juristen, die in der Regel Berufsrichter sind, und zwei Beisiczern, die auf Vorschlag der Vereinigungen der Hauseigentümer und der Mieter bestellt worden waren. Dem Vorsizzenden, im konkseten Fall ein Berufsrichter, kam bei einem Patt die ausschlaggebende Stimme zu. Da der Beschwerdeführer ón Kollekiivvertrag grundsätzlich angriff, hielt der Gerichtshof dic Bereiligung der beiden ehrenamtlichen Richter, die selbst diesem korporativen System angehören, für unzulässig, ohne dass es auf die Mitwirkung der beiden Berufsrichter ankämé2.

I EGMR, $A /$ Iss (Langborger).

2 EGMR, A/I 5 , $\$ 35$; kril diss. op. Pinhciro Farinha, diss. op. Pctriti/Valticos. 
Mir dieser Begründung könnten aber auch Arbeitnchmer oder Arbeitgeber, die keiner Tarifpartei angehören, die Zuständigkeit der paritätisch besetzten Spruchkörper der Arbeitsgerichtsbarkeit für unzulässig erklären, zumal die Tarifflucht und die Kritik am System der Kollektivverträge in den letzen Jahren zunehmen'. Da es nicht möglich iš diese Außenseicer-Konstcllationen abstrakt aus der Zuständigkeit der entsprechenden Gerichtsbarkeic herauszunehmen, wcil die Besetzung des Spruchkörpers nicht von einer Eigenschafr des Klägers oder der Begründung der Klage im konkreten Fall abhängig gemacht werden kann, stelit das Urteil letztlich das Interessengruppen-Prinzip bei der Auswahl chrenamtlicher Richcer insgesamt in Frage. Auf diese weirreichenden Konscquenzen hat die abweichende Meinung der Richter Pettiti und Valricos zurreffend hingewiesen.

\section{Auswablkriterien chrenamtlicher Richter}

Das hier untersuchte Problem der struknurellen Unpartcilichkeit stelit sicls aber auch bei anderen spezialisierten Gerichten, in denen ebenfalls ehrenamtiche Richcer tätig sind, die nichr die Allgemeinheir vertreten, sondem aufgrund besonderer persönlicher Merkmale ausgewählt werden, wie z. B. in der Sozial-, Berufs- oder Disziplinargerichtsbarkeit. Der Einsatz von ehrenamtlichen Richtern hat zwar keine ausdrückliche Basis im Grundgeserz, nach Auffassung des Bundesverfassungsgerichtes bestehen jedoch keine grundsätzlichen Einwände gegen die Beteiligung ehrenamtlicher Richter, da das Grundgeserz diese Rechtstradition anerkennet. Dementsprechend gibt es im deurschen Gerichtsverfassungsrecht eine Vielzahl von Spruchkörpern, die nicht ausschließlich aus Berufsrichtern bescehen'. Insbesondere in spezialisicrten Spruchkörpern der Verwaltungsgerichrsbarkeiten spielen ehrenamtliche Richter eine wichrige Rolle.

Dabei ist entsprechend der unrerschiedlichen Auswahlkriterien zwischen mehreren Typen von ehrenamtlichen Richtern zu unrerscheiden ${ }^{6}$. Es gibt einerseics das 》Jedermann-Prinzip a des Laienrichters, der als Vertrezer der Allgemeinheiz fungiert. Hierzu gehören die größten Gruppen, die Schöffen bei den Strafgerichten ${ }^{7}$ und die ehrenamtlichen Richter der Finanz- ${ }^{\delta}$ und der allgemeinen Verwaltungsgerichtsbarkeit?.

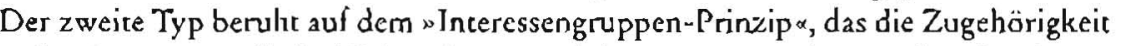
zu bescimmten gesellschaftlichen Gruppen als Vorausserzung der Berufung bestimme. Er umfasst neben der Arbeits- und Sozialgerichisbarkeit ${ }^{\circ}$ auch einige spezialgesetzlich eingerichtece Spruchkörper wie die Disziplinargerichee, die Fachkammern für Personalvenrecungsangelegenheiten, die Flurbereinigungsgerichte und die Land-

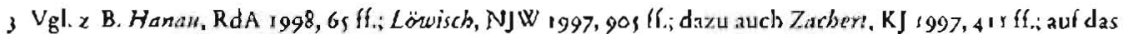
Außenscitcrproblem als Argument gegen die Laicnberciligung verweist ausdrücklich Windel, ZZP 112 (1999), 29) If., 308 !

4 BVEr \{GE 14, 56,$73 ; 26,186,198 ; 27,111,319 f ; 42,206,208 f, 48,300,31$ ?

5 Vgl. die Aufzählung bei Schsnids. Ränesch. Deursches Richtergesetz, s. Aufl, $\$ 44$ Rn. s.

6 Vgl. Rüggebcrg, VerwArch 6r (1970), 189 if., 197: Riedel, Das Pastulat der Unparteilichkeit des Richers, S. 98 f.; Schmidt-Rantsch (Fn.5). \$44 R 1. 4 f.; Heyde, in: Bendi/Maihofer/Vogel (Hrsg.), Handbuch des Verfassungsrechts (HdbVerfR), 2. Aufl. \$33 Rn. 86; Barbey, in: Iscnsce/Kirchhof (Hrsg), Handbuch dcs

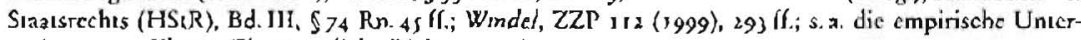
suchung von Klausa, Ehrenamiliche Richecr, passim.

$7 \mathrm{Vgl}$. Rennig, Die Entscheidungsfindung durch Schollen und Bcrulsricher in rechalicher und psychologischer Sicht, passim; Casper/Zeisel (Hrsg.), Der Laicnrichter im Serifproxe.ß.

8 Vgl. Reim, DRiZ r $1992,{ }_{39} \mathrm{ff}$.

9 Vgl. Schiffmann, Dic Bedeutung der chrcnamelichen Richter bei Gerichten der allgemeinen Verwalıungsgerichtsbarkcit, S. 79 il.; Ziekow, in: Sodan/Zickow (Hrsg.), VwGO, \$ 19 Rn. 2ff.; Schnellenbaci. ES Menger, S. 34 Iff., $3<2$ ff.

10 Oseheimer/Wiegand/Hohmann. Der chrenameliche Richer beim Arbeits- und Sozialgericht, 8. Aun, S. i ff., beronen allerdings auch den Gesichtspunki des Sachuersiandes. 
wirtschaftsgerichte. Als drittes wird das "Sachverstands-Prinzip" genannt, dem die Handelsrichter, die ehrenamtlichen Richter der Kammern für Handelssachen, zugeordnet werden. Allerdings ist bei ihnen nicht nur die leitende Funktion in einem Unternehmen Voraussetzung für die Ernennung ( $\$ 109$ Abs. I Nr. 3 GVG), außerdem ist nach $₫ 108$ GVG ein gurachulicher Vorschlag der Industrie- und Handelskammern erforderlich, so dass hięr ebenfalls ein korporatives Element zu finden isc"

Während̀ die die Allgemeinheit vertretenden ehrenamtlichen Richter kein Problem der strukturellen Unparteilichkeit aufwerfen, ist die Problematik bei den anderen beiden Erscheinungsformen offenkundig. Sachlich spezialisierte Spruchkörper, die mic Richcern besetzc werden, die einer besrimmten Gruppe der Bevölkerung entstammen, werden vom Bundesverlassungsgericht jedoch für grund́säczlich zulässig gehalten. Ob und in welchem Umfang sic zugezogen werden, stehe im Ermessen des Gesetzgebers'2. Die Grenze für ihre Bcteiligung hänge von den Umständen ab']. Es anerkennt auch keine Pflicht, $\mathrm{da} ß$ jeder gerichtliche Spruchkörper mit mindestens einem Berulsrichter beserzt sein muß. Viclmelir hat es die Regelung des $\$ 94$ Abs. I S. I BRAO gebilligt, daß dic Anwalrsgerichce als erste Instanz der Ehrengerichtsbarkeit ausschließlich mic Anwälıcn beserze sind'". In diesem Fall isc allerdings atypischerweise gesichert, daß die ehrenamtlichen Mirglieder die Befahigung zum Richteramt besitzen, da sie Voraussetzung für die Zulassung als Rechtsanwalt ist.

Eine andere Form der Gefăhrdung der Unparteilichkeic eines Gerichts stellt die gleichzentige Ausübung von exekutiven und judikativen Funkrionen dar. Das in Arr. 20 Abs. 2 GG verankerte Prinzip der Gewaltenteilung verlangr, daß die Rechtsprechung durch besondere Organe ausgeübr wird, die von den Organen der Gesetzgebung und der vollziehenden Gewalt verschieden sind. Deshalb müssen die Gerichte nicht nur organisatorisch hinreichend von den Verwaltungsbehörden geurennt sein, die richterliche Neutralicät darf auch nicht durch eine mit diesem Grundsatz unvereinbare persönliche Verbindung zwischen Ämrern der Rechtspflege und der Verwaltung oder der Legislarive in Frage gesteilt werden's. Dieser Grundsatz ist bei den Berufsrichrern weirgchend unproblematisch, da ihre Neutralität durch die Anstellung auf Lebenszeit und die in Art. 97 Abs. 2 GG verbürgre Garantie persönlicher Unabbängigkeit gesichert wird. Außerdem ergibt sich aus $\$_{4}$ i.V.m. $\$ 2 D R i G$, daß Berufsrichter nicht gleichzeitig Aufgaben der rechtsprechenden und der vollziehenden Gewalt wahrnehmen dürfen.

Anders stellr sich die Situation dagegen bei den ehrenamclichen Richtern dar. Auch ihnen wird zwar durch eine feste Amzszeit und durch $\$ 4{ }_{44} D R i G$, der vorsichs da $\$$ sic nur durch ein Gericht abberufen werden können, die grundgeseczlich erforderliche persönliche Unabhängigkeir gewährleistet ${ }^{16}$. Es gibr jedoch keine ausnahmslose Inkomparibilität zwischen der Wahmchmung einer exekuriven Funkrion und der'Tärigkeit als ehrenamelichem Richter in einem Spruchkörper im Bereich der verschiedenen Verwaltungsgerichtsbarkeiten. Dic gesetzlichen Regeln lassen vielmehr in vielen Fälien Übcrschneidungen zu.

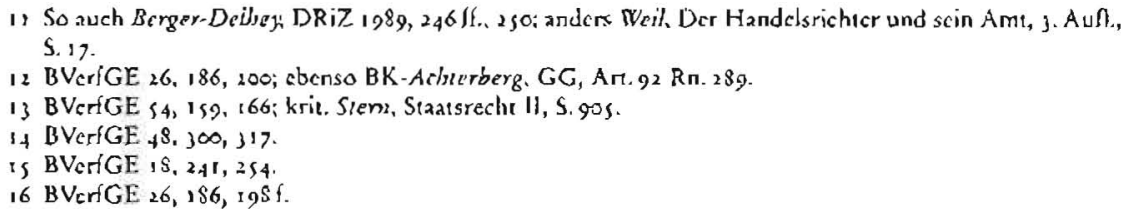


Der Europäische Gerichtshof für Menschenrechte wendete im Fall Langborger das Gebot der Unparteiluchkeit an, das eine der drei zentralen organisatorischen Anforderungen darstell, die von der Europäischen Menschenrechtskonvention für ein Gerichr festgelegt werden. Hierbei sind zwei verschiedene Aspektc zu unterscheiden. Auf der einen Seite wird als Problem der Unparteilichkeit die Fragc personenhezogener Ausschluß- und Befangenheitsgründe bzw. des Ausschlusses wegen Vorbefassung in einer anderen Funktion behandelt"7, die auf besondere Beziehungen eines Richters zum konkreten Prozessgegenstand verweist. Hier ging es aber nicht um die Person eines bestimmten Richters, sondern um das Besetzungsverfahren als solches. Es stellte sich das Problem einer strukrurellen Inkomparibilität, einer vom Einzelfall unabhängigen generellen Befangenheit. Für diesen speziellen Aspekt der richterlichen Unparteilichkeit passt die vom Straßburger Gerichrshof in einigen Urteilen verwendece Unterscheidung zwischen einem subjektiven und einem objektiven Aspekt der Unparteilichkeit ${ }^{18}$ nicht, zumal er diesen beiden Begriffen bisher keine klaren Konturen gegeben hat.

Um eine eindeurige Zuordnung der Problemkonstellacionen zu crmöglichen, wird hier deshalb vorgeschlagen, in denjenigen Fällen, in denen sich ein abstrakter Rollenkonflikt ergibr, der an Umständen anknüpit, die nicht nur den konkreten Streicgegenstand berühren, von einem Problem der strukturellen Unabhängigkeit zu sprechen. Sie betrifft neben der Zulässigkeit der Zugehörigkeit zu einer bestimmten gesellschafrlichen Gruppe auch die Frage, ob sich durch die gleichzeirige Wahrnehmung einer exekuriven Funktion eine generelle Unvereinbarkeit mit der justiziellen Funktion ergibu. Es geht jeweils um die generelle Frage, ob es zulässig, daß Richter aus einem eingeschränkten Personenkreis ausgewählt werden dürfen, um über spezielle Sachgebiece zu entscheiden.

Da die Unparteilichkeit unmittelbar mic der Gerichtsqualität verknüpft ist, stellı sich die Probiematik strukcureller Inkompatibilitäten nicht nur im Anwendungsbereich der EMRK, sondern auch auf verfassungsrechtlicher Ebene. Das Grundgesecz enthält zwar keine explizite Garantie der Unparteilichkeit der Gerichre. Das Bundesverfassungsgericht vererict jedoch in ständiger Rechtsprechung die Auffassung, daß es der richterlichen Tätigkeit wesentlich sei, daß sie von einem nichtbeteiligten Dritten ausgeübt werde'9. Zum Teil wird auch der Begriff der richterlichen Neutralitäı im Sinne von Unvoreingenommenheit - verwendet ${ }^{20}$. Auf der Grundlage dieses Prinzips hat die Rechrsprechung eine Reihe von Entscheidungen zu dem Problem gencreller Unvereinbarkeiten getroffen, die jedoch keine klare Linie erkennen lassen. Weder in der Rechrsprechung noch in der Literanur ist bisher der verfassungsrechtliche Standort des Problems der Unparteilichkeit und die Reichweite der sich daraus ergebenden Anforderungen systematisch herausgearbeitet worden. Während die Pluralisierung der Exekutive inzwischen breit diskusiert wird"', iss das gleiche Phänomen inncrhalb der Judikative mit den sich daraus ergebenden Folgeproblemen bisher praktisch unbeachret geblieben.

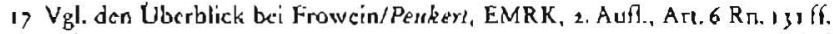

$18 \mathrm{Vgl}$. EGMR, A/s3 (Piersack). \$30= EuGRZ 1985, 301, 303; A/80 (Campbell, Fell), \$85 = EuGRZ igs s, 534, SA1; A/292-B (Debled), 36; s. a. Miehsler/Vogley, IntKommEMRK, Art. 6 Rn. 301; Dör, in: Sodzn/ Ziekow (Hrsg), VwGO, EVR Rn. s8o; Villiger, Handbuch der Europasschen Menschenrechrskonverion (EMRK), Rn. 414 亿\}.

19 BVerGE 3, 377, 3S1; 26, 186, 198; 60, 173, 214;87,68, 85.

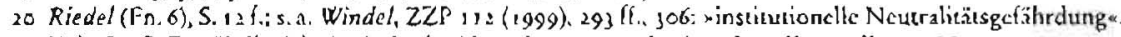

21 $V_{g} l . G r o ß$, Das Kollegialprinzip in der Verwaitungsorganisation. S. 45 ff, 130 ff. m.w.N.
} 
Im Folgenden wird untersuchs, ob es sich beim Gebot der scrukcurcllen Unpartei-

lichkeit um ein eigenständiges, allgemeines Prinzip handels, das einen Mindeststandard festlegt, dem jedes Gericht genügcn muß. Nur in diesem Fall würde es über den Anwendungsbereich des Art. 6 Abs. I EMRK hinausreichen, der nach seinem Wortlaut auf zivilrechtliche Ansprüche und Verpflichoungen sowie strafrechtliche Anklagen beschränkt ist, und der auch in der wciren Auslegung der Straßburger Rechtsprechung nur Teilbereiche der Zuständigkeir der Vcrwaltungsgerichtsbarkeit etfasst". Wenn es sich um ein Wesensmerkmal jedes Gerichrs handelt, mußte es ebenso aus dem deurschen Verfassungsrecht, insbesondere Arr. 92 GG, abzuleiten scin.

Zu diesem Zweck wird zunächst die einschlägige Rechtsprechung analysiert (II.). In einem Exkurs wird auf dic Bedeurung der Unparteilichkeit bei der Bestimmung der vorlageberechrigcen Gerichte nach Art. 234 EG eingegangen (III.). Anschließend wird das Verhältnis zwischen dem Erfordernis der Unparteilichkeit und dem allgemeinen Prinzip der richcerlichen Unabhängigkeit untersucht (IV.). Hieraus ergibı sich dic Frage nach Mechanismen zum Schurz der Unparteilichkeit, die eng mit der Legitimation der ehrenamtlichen Richcer verknüpft ist (V.).

\section{I1. Überblick über die Rechisprechung}

\section{Europäischer Gerichishof für Menschenrechte}

Das erste Verfahren, in dem der Straßburger Gerichtshof Gelegenheit hatte, das Erfordernis der Unparteilichkeir des Gerichts nach Art. 6 Abs. I EMRK zu präzisieren, betraf die belgische ärztliche Beruísgerichtsbarkeit" ${ }^{23}$. Der Gerichushof lehnte in Bezug auf diesen Punkt entgegen dem Vorum der Kommission eine Konventionsverleczung ab. Er beschränkte dabei seine Prüfung auf die zweite Instanz des disziplinarischen Verfahrens. Der zuständige Spruchkörper bestand je zur Hälfte aus Ärzten und aus Berufsrichtern, zu denen auch der Vorsitzende zählte, dessen Vorum bei Stimmengleichheit ausschlaggebend war. Die äıztichen Mirglieder wurden von den Provinzräten, die die ersce Instanz bilden und ausschließlich mit Ärzten beseczr sind, gewählc. Die Bcdenken, die sich daraus ergaben, daß die ärzrlichen Mirglieder aus dem Kreis ihrer Berufskollegen gewählt wurden, verwarf der Gerichtshof, da durch das leichte Ubergewicht der Berufstichrer eine ausreichende Vorsorge für die Unparteilichkeit des Gerichts gecroffen sei.

In einem anderen Fall verneinte der Gerichtshof die Vereinbarkeit der Zusammenseczung der als Gericht in Grundsücksangelegenheicen fungierenden Tiroler Landesgrundverkehrsbehörde mit Arr. 6 Abs. I EMRK ${ }^{14}$. Wie bercits zuvor"s akzeptierse der Gerichtshof zwar grundsärzlich, daß auch Laien und Angehörige der Exekucive Mitglieder eines Gerichrs scin können. Er rügte aber, daß der Berichterstatter des Geriches ein Bcamter der Landesbehörde war, dessen Vorgesetzter das Verfahren selbst durch Berufung in die zweite Instanz gebracht hatte. Deshalb musse der

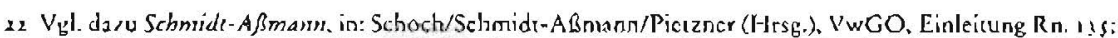
Dorr (Fn. 18), EVR Rn. 574 ff, ausführlich Grabenwarter, Verfihrensgarantien der Verwaleungsgerichisbarkci, S. 3 ) ifl.

2) $E G M R, A / 43=E u G R Z$ ig $\$ 1$. s s I (Le Compte, Van Leuven, De Mcyere); bestatigt in EGMR, A/29z-B.

${ }_{24} \mathrm{EGMR}, \mathrm{A} / \mathrm{s}_{4}$ (Sramek); wcilergehend conc. op. Ganshof van der Meersch/Evrigenis; krit. dis5. op. Evans/ Gersing

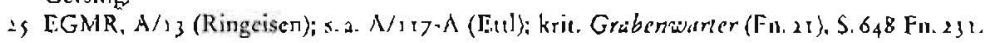


Beamte als Vertreter einer Partei, der beteiligten Bchörde, erscheinen. Die Tatsache, daß ihm in konkreten Verfahren keine Weisung erteilt werden könne, reiche nicht aus, den Anschein der fehlenden Unabhängigkeis zu beseitigen. Hierbei unterschied der Gerichishof nicht zwischen den beiden Merkmalen der Unabhängigkeit und Unparteilichkeit, er prüfte jedoch der Sache nach abstrakte Fragen der personellen Besetzung des Spruchkörpers, die aufgrund der engen Verzahnung mit den Zuständigkeiten der Behörde einzelfallunabhängig waren.

In einer jüngeren Entscheidung ${ }^{26}$ stand die ärztliche Berufsgerichtsbarkeit in Frankreich auf dem Prüfstand. Die Beschwerdeführer wehren sich gegen eine Disziplinarstrafe, die von einem Regionalrat der Ärztckammer verhängt und von der Dišiplinarsektion des Nationalen Rates der Ärztekammec, in abgemilderter Form, bestätigt wurde. Sie war verhängt worden, weil die Beschwerdeführer beim Betrieb eines Nodalldienstes gegen das standesrechtliche Werbeverbot verstoßen hatten. Der Gerichtshof folgte der Auffassung, daß in diesem Fall kein unparteiliches Gericht entschieden habe. Da die Ärzcekammern sclbst Norfalldienste betrieben, komme es aufgrund der Tarsache, daß die ärzrlichen Beisirzer, die auch in der zweiten Instanz die Mehrtheit der Richter stellen, durch die Ärztekammern gewählt werden, zu einem Interessenkanflikt. Den Einwand der französischen Regierung, dass die Beschwerdeführer die Möglichkeit gehabt hätten, den Staatsrat, das höchste Verwalrungsgericht, im Wege der Revision anzurafen, ließ der Gerichtshof nichs gelten, da eine Rückverweisung der Entscheidung an die Disziplinarsektion nichı auszuschließcn gewesen sei, so daß es sich nicht um ein adäquares Rechrsmitrel gehandeir habe.

Als vorläufiges Fazic dieser Rechtsprechung kann festgehalten werden, daß die Beteiligung ehrenamtlicher Richrer, auch wenn es sich um Verwaltungsangehörige handelt, vorn Straßburger Gerichtshof als grundsätzlich zulässig angesehen wird ${ }^{77}$. Auch eine Melurheic von Berufsrichtern im Spruchkörper ist nicht in jedern Fall nowwendig, um die Anforderungen des Arr. 6 Abs. I EMRK zu erfüllen. Es muß aber sichergestcllt sein, daß das Gericht nicht durch die Bindung an Gruppeninteressen voreingenommen ist. Zumindest im leczrinscanzlich entscheidenden Spruchkörper muß die Mehrheit seiner Mitglieder hinreichend distanziert gegenüber den Beteiligten des Prozesses sein. In jedem Fall unzulässig ist es, daß ein Bediensteter einer in Parteistellung bereiligren Behörde als Richter fungier.

\section{Bundesverfassungsgericht}

Die beiden vom Straßburger Gerichishof als Probleme der strukturellen Unparteilichkeit behandelten Konstellationen finden sich auch in der deutschen Rechtsprechung wieder. In der einen Fallgruppe geht es darum, dass Richeer aus einer bestimmten gesellschafrlichen Gruppe ausgewählt werden und deshaib Zweifel an ihrer Unvereingenommenheit geltend gemache werden (a). In der anderen Fallgruppe ist die Zulässigkeit von Überschneidungen zwischen exekutiven und richterlichen Funktionen strittig (b).

\footnotetext{
26 EGMR 1998 -III, S. 1009 (Gautrin u.a).

27 Ebenso q. Dijk/van Hoof, Theory and Practice of the European Convention on Human Righrs, 2. Auf. S. 338: Tomuschat, FS Redeker, S. 273 [f., 28 \}f.
} 
Das Bundesverfassungsgericht hat bisher in allen Fällen die geltenden Auswahiregeln des Gerichisverfassungsrechrs für verfassungsmäßig erklärc. Sowohl die Heranziehung von Kassenärzten als ehrenameliche Beisitzer in den Kammern bzw. Senaren für Kassenarztrecht der Sozialgerichtsbarkeit ${ }^{2 k}$ als auch die Beteiligung von landwirtschaftlichen Beisitzern in gundstücksverkehrsrechtichen Angelegenlyciten ${ }^{29}$ wurden von ihm akzepriert. Es sei nicht anzunehmen, daß sie in aller Regel bestrebt seien, einseitige, nicht am Wohl der Allgemeinheit orientierte Interessen durchzusetzen. Daß Kassenärzte durch Entscheidungen in Honorarstreitigkeiten entfernt mitbetroffen sein könnten, stelle den Grundsatz der richterlichen Neurralität noch nicht in verfassungswidriger Weise in Frage. Das Gericht räumte allerdings ein, daß die Wahrscheinlichkeir eines Falles der Befangenheit in diesem Bereich höher sein könne als in der allgemeinen Gerichrsbarkeit. Dafür genügten jedoch die Vorschriften über die Ablelınung der Gerichtspersonen ${ }^{30}$. Auch bei den Landwirten liege keine unzulässige generelle Interessenbindung vor.

\section{b) Die Parteilicbkeir exekutiver Funktionsträger}

In bezug auf die zweite Problemkonstellation hat das Bundesverfassungsgericht differenzierende Kriterien angelegt. Die Verbindung von Richter- und Verwaltungsame auf Gemeindeebene in den inzwischen abgeschafften Gemeindegerichten in Baden-Wüntemberg, die für bescimmte zivilrechtliche Streitigkeiten zuständ́ig waren, war mit dem Argument, daß die Gemeinde vor den Gemeindegerichcen aufgrund deren beschränkter Zuständigkeit nicht als Partei auftreten konnte, als zulässig angesehen worden ${ }^{31}$. Eine konkrete Überschneidung der exekutiven und der judikativen Funktionen war foiglich ausgeschlossen.

Die Bestimmungen des rheinland-pfälzischen Ärzcekammergesetzes über die Berufsgerichte wurden dagegen wegen Verstosses gegen Art. 20 Abs. 2 GG für verfassungswidrig erklärt, weil sie nicht ausschlossen, daß Angestellte der Kammer und der Vertreterversammlung zum Richteramt berufen wurden ${ }^{32}$. Bei den Angestellten wurde darauf abgestellh daß sie einerseits den Weisungen des Vorstandes unterworfen seien und andererseits als Richter über dessen Antrage entscheiden müßten. Einen Rollenkonflikt sah das Gericht ebenfalts bei den Mitgliedern der Verreterversammlung, da diese als wichtigstes Organ der Kammer die Grundsätze für deren Tätigkeit maßgeblich bescimme. Deshalb erscheine ein Vertreter bei der Überprüfung der Rechtmäßigkeit der Beschlüsse der Kammer der Natur der Sache nach selbst als Partei.

Für zulässig wurde andererseits erklär, daß Mitglieder der Vollversammlung der Landwirtschaftskammer in Rheinland-P\{alz, die enrgegen dem üblichen Wortsinn ein gewähltes Vertrecungsgrenium ist, als Beisitzer der Landwirtschaftsgerichre in Angelegenheiten des Grundstücksverkehrs entscheiden'". Da die Vollversammlung in einzelnen Genehmigungsverfahren nur in Form einer Anhörung beteilige werde, und

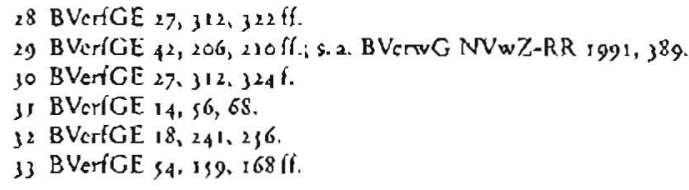


auch dies nur, wenn ihnen grundsätzliche Bedeurung zukomme, handele es sich nicht um eine nsubscantiell verwalcende Tärigkeit «. Außerden seien die Organe der Landwirtschaftskammer zu einer Aufgabenwahrnehmung im Interesse der Allgemeinheit verpflichtet. Eine generelle Sachbefangenheir liege deshalb nicht vor. Ein Ausschluß sei vielmehr nur erforderlich, soweit der landwircschafdiche Beisiczer im Einzelfall als Mirglicd der Vollversammlung mir der nunmehr von ihm richterlich zu überprüfenden Genehmigungsversagung befaßt worden war. Hierfür scien die Vorschrifren in $\$\{4$ Abs. 2 VwGO, $\$$ I $\mathrm{Abs}$. 2 FGO und $\$ 60$ Abs. 2 SGG über den Ausschluß wegen Vorbefassung entsprechend anwendbar ${ }^{34}$. Dagegen sah das Gericht bei den Vorstandsmitgliedern der Kammer eine erhöhre Gefahr eines generellen Pflichrenwiderstreits und erklärte das Fehlen einer Vorschrift über ihren Ausschluß wegen Verstoßes gegen die Grundsăcze des Art. 20 Abs. 2 und des Art. 92 GG und gleich. zeitiger Unvereinbarkeit mit Art. 19 Abs. 4 und Arr. 101 Abs. 1 S. 2 GG für verfassungswidrig".

Auch in der Rechtsprechung der obersen Bundesgerichte sind mehrfach vergleichbare Konstellationen behandelt worden. Keine Bedenken wurden dagegen erhoben, daß aktive Beamte der Fachverwaltung in den Flurbereinigungsgerichten als Beisizzer rätig sind ( $\$ S 138$ Abs. I S. I, I 39 Abs. I S. 2 FlurbG), ihre Mirwirkung könne jedoch nur in Gerichten eines anderen Bundeslandes erfolgen ${ }^{16}$. Als zulässig orurde auch angesehen, daß ein aus der beklagten Fachverwalung ausgeschiedener Beamter als Beisizzer fungier ${ }^{37}$.

Das Bundessozialgericht hat aus Art, 20,92, 97 A.bs. I GG abgeleitet, daß Bedienstete der Versorgungsverwairung niche Mirglieder eines Spruchkörpers sein können, der Angelegenheicen aus ihrem Sachgebiec behandel $t^{\prime 8}$. Ein Ausschluß im Einzelfall nach $\$ 60$ Abs. 2 SGG wegen vorheriger Mirwirkung in einem Verwalcungsverfahren wurde ausdrücklich als nicht ausreichend angesehen'?. Die Ausnahme des $\$ 17$ Abs. 4 SGG für Geschäftsführer der Kassenärzclichen (Kassenzahnärztlichen) Vereinigungen und ihre Stellvertreter, die Mitglieder der Kammern für Angelegenheiten des Kassenarztrechts sein können, wurde dagegen in späteren Entscheidungen des Bundessozialgerichts mit ihrer besonderen Fachkunde gerechtfertigt ${ }^{\circ}$.

Ungeachter einiger Ungereimtheiten in der Detailabgrenzung belegen diese Entscheidungen, daß den Gerichten das Problem fehlender strukrureiler Unparteilichkeir, das sich aus cinem generellen, niche nur auf das konkrete Verfahren bezogenen Rollenkonflikt ergeben kann, durchaus beoußt war. Es ist deshalb zu kurz gegriffen, wenn diese Fälle pauschal aus dem Problem der Unparteilichkeit ausgeklammert werden, indem auf den fehlenden Fallbezug eines solchen wallgemeinen Interesses $*$ hingewiesen wird ${ }^{41}$. Parteilichkeir kann nicht allein auf Fälle einer unsachlichen inneren Einstellung zu Beteiligten oder zum Gegenstand des konkreten Verfahrens begrenzt werden ${ }^{21}$.

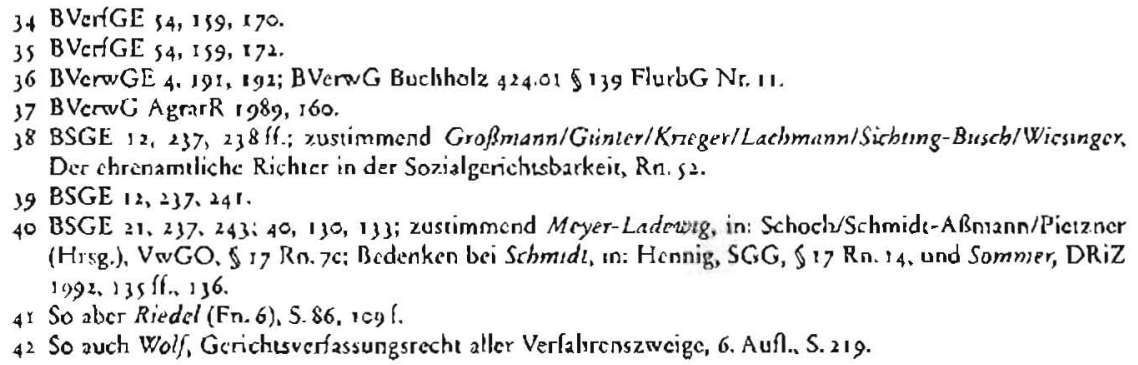


Eine weitere Dimension erhält das Problem der Unparteilichkeit, wenn man die im EG-Recht entwickelten Voraussetzungen für die Vorlageberechtigung nach Art. 234 EG (bisher Art. I77 EGV) einbeziehr. Da diese Vorschrift selbst keine Definition enthält, hatte sich der Gerichtshof der Europäischen Gemeinschaften in mehreren Entscheidungen mit der Frage auseinanderzusetzen, welche Bedingungen eine nationale Srelle erfüllen muß, damit sic als Gericht im Sinne des Gemeinschaftsrechts anerkannt werden kann. Einer der Gesichespunkte, der dabei eine Rolle spielt, ist die Unparteilichkeit, wobei nur objekıive Aspekre berücksichtigt werden können, da die Entscheidung über die Vorlageberechtigung aufgund von abstrakten, fallunabhängigen Kriterien gerroffen werden muß.

Der Gerichtshof kann sich jedoch nicht ohne weiteres an der jeweiligen rationalen Kacegorisierung orientieren, sondern muß autonome europarechcliche Abgrenzungskricerien entwickeln. Generalanwalt Gand betonte bereits im Jahr 1966 in dem Verfahren, das zum ersten grundlegenden Urteil zu dieser Frage führte, daß der gemeinschaftsrechtliche Gerichtsbegriff nicht von historischen Zufälligkeiten oder unterschiedlichen Rechtsauffassungen über die Gerichts- und Verwaltungsorganisation der Mitgliedstaacen abhängen könnet3. Bei der Analyse der Rechtsprechung ist allerdings zu beachten, daß siclı der EuGH durch eine großzügige Auslegung selbst den Weg öffnet, in einer möglichst großen Zahl von Fällen die Vorlagefrage zu behandeln, um dadurch mögliehst weitgehend seinen Einfluß auf die Durchsezzung des Gemeinschaftsrechts zu sichern ${ }^{44}$.

Die Anerkennung der Vorlageberechtigung durch den EuGH seczt die Erfüllung von sechs Kriterien voraus: Die nationale Stelle muß weisungsunabhängig sein, eine gesetzliche Grundlage haben, ständig eingerichtet sein, eine obligatorische Gerichtsbarkeit ausüben, ein streitiges Verfahren durchführen und nach Rechtsnormen entscheiden"s. Nichr norwendig ist hingegen, daß das Gericht Teil der staatlichen Organisation ist. Der Gerichtshof anerkannte die Vorlageberechtigung z. B. auch im Fall des niederländischen Strcitsachenausschusses für Angelegenheiten der allgemeinen Medizin, einem Gremium der privatrechtlichen Königlich-Niederländischen Gesellschaft zur Förderung der Medizin, die aufgrund einer Verordnung für die Anerkennung als praktischer Arıt zuständig ise ${ }^{\circ}$. Die Mitglieder des Ausschusses werden nach der Geschäftsordnung der Gesellschaft auf fünf Jahre ernannt. Drei Mitglieder werảen durch die niederländischen Medizinischen Fakulcäten, drei durch den Vorscand der Gesellschaft und drei - daruncer der Vorsiczende, vorzugsweise ein hoher Richeer - durch die Minister für Hochschulunterricht bzw. für die öffentliche Ge-

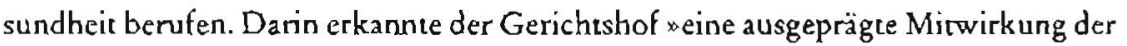
niederländischen Behörden «. Auch der Generalanwalt konstatierte die Garantie einer gewissen Unabhängigkeit durch die Zusammensetzung des Ausschusses ${ }^{4}$.

Ein dänisches Schiedsgericht, das durch Tarifvertrag errichcet wurde, ist ebenfalls als vorlageberechcigt anerkannt worden. Der Gerichushof hielt es für ausreichend, daß das dänische Arbeitsgerichtsgesetz einen Vorrang tarifverraglicher Streitentscheidungsmechanismen vorsieht und die Zusammensetzung des Gerichtes regelt, namentlich die $Z$ ahl der von den Parteien zu besrellenden Mitglieder sowie die Auswahl

43 EuGHE 1966, $\{83,611$ (Vxasscn-Göbbcls).

14 Calogeroposlas, RTDE 1987. 3s ff. 39; Gröning, ZIP 1998, $37016 . .371$.

4) Dasses, in: ders., Handbuch des EU.Wiruschaísrechts, P.II. Rn. 44; Dör (Fn. 18), EVR Rn. 225 ; Ehlen, in: Schoch/Schmidt-A Bmann/Picizner (Hrsg), VwGO, Anli $\$ 40$ An. 177 EGV Rn. 13.

${ }_{46} 6$ EuGHE 1981,2111, 2326/f. (Brockmeulen).

4) EuGHE 19S1, 2311,2339 . 
des Obmanns, falls sich die Parteien hierüber niche cinigen ${ }^{48}$. Die Mchrzahl der Mitglieder wird also in jedem Fall ohne Mirwirkung staatlicher Stellen ausgewählt.

Dagegen verneinte der Gerichrshof die Vorlageberechtigung des Direktors der luxemburgischen Steververwaltung, obwohl er in der Srellungnahme der nationalen Regienung als Gericht anerkannt worden war. Der Generalanwalt wies darauf hin, daß die Unabhängigkeit des Richters gegenüber den Streitparceien ein wesentliches Merkmal sei, das bei einem dem Finanzminister unterstellten Teil des staatlichen Machrapparaces niche erfüllt sein könnełs. Der Gerichtshof schloß sich dieser Auffassung an und beconte, daß es zum Wesen des gemeinschafissechrlichen Gerichtsbegrifffs gehöre, daß die Einrichrung gegenüber der Stelle, deren Entscheidung Gegenstand der Klage sei, die Eigenschaít eines Dritten habe ${ }^{\text {s. }}$. Dies sei bei der offensichrdichen instirutionellen Verbindung zur Steuerverwaltung nicht der Fall. Außerdem sei der Direkcor bei einer beim Staassrat als nächsthöherer Instanz eingereichren Klage in Steuersachen selbst Partei des Verfahrens.

Einen großzügigen Maßstab legte der Gerichtshof in der Enrscheidung über die Vorlageberechtigung des Vergabeüberwachungsausschusses des Bundes an, die noch auf der Grundlage der haushaltsrechrlichen Regelung erging". Der Generalanwalt hatte schwerwiegende Bedenken gegen seine Unabhängigkeit geäußerr ${ }^{\prime 2}$. Er monierte, daß die beamteren Mizglieder des Ausschusses gleichzeirig für das Bundeskartellamt tätig scien und es keine Regelung gebe, die ihre Unabsetzbarkeit garantiere. Außerdem verneinte er die Eigenschaft des Ausschusses als Dritter, da er Teil des Bundeskartellamtes und damit der Verwalsung sei, aber über die Vergabe öffentlicher Aufträge, also Streitigkeiren zwischen der Verwaltung und Bürgern zu entscheiden habe. Er nahm dabei Argumente auf, die auch in der deurschen Diskussion gegen die Gerichrsqualität der Uberwachungsausschüsse angeführc worden waren"3. Bemängelt wurde dabei ebenfalls die fehlende organisarorische und funktionelle Unabhängigkeir. Problematisch war insbesondere, daß für die beamteten Mitglicder keine festc Amrszeit und keine Regelung über die Abrufbarkeit festgelegt worden war ${ }^{34}$. In der Begründung des Gesetzentwurfs der Bundesregierung wurde das Gremium der im deutschen Recht bisher unbekannten Kategorie der "gerichtsäquivalenten Instanz" zugeordnes", die zwar die Kriterien des Art. 177 EGV erfülle, aber kein Gericht im Sinne des Art. 92 GG sei. Der Gerichtshof bejahte jedoch die Gerichtseigenschaft und stützre sich dabei u. a. auf $\$ \$ 7 \mathrm{C} \mathrm{HGrG}$, der nicht nur die sachliche Unabhängigkei, sondern durch den Verweis auf die wesentlichen Vorschriften des Deurschen Richtergeserzes auch die persönlishe Unabhängigkeit der Mitglieder der Kammern garanuere ${ }^{16}$. In bezug auf die Unparteilichkeit der Mitglieder hielt er es für ausreichend, dass sie nicht mir Fällen befasst werden dürfen, bei denen sie selbst an der Vergabeentscheidung mitgewirkr haben oder bei denen sie Bieter oder Inceressenvertreter von Bietern sind oder waren.

Zusammenfassend kann man festhalten, daß die Unparteilictkeit zwar zu den Merkmalen des Gerichtsbegriffes nach Art. ${ }_{234}$ EG gehört, daß dieses Erfordernis aber großzügig gehandhabt wird. Es wird niche verlange, daß die Richter von den Parteien

$4^{3}$ EuGHE 1989, 3199, 3224 (Danfoss).

49 EuGHE. 1993, 1277, 1290 (Corbiau).

10 EuGHE 1993, 1277, 1304.

s1 Zur zwischenzcitlichen Ncurcgclung im GWB vgl. z. B. Pietzcker, ZHR 162 (r998), \{27 If.; Byok. NJW 1998,2774 ft.

s2 EuGHE r997-I, 4961, 4976 (f. (Dorsch-Consult).

\$3 Vgl. Sclimidt-Aßmann (Fn.22), Einleitung Kn. 2s; Ehlers, in: Schoch/Schmidt-Aßmann/13ictzner (Hrsg),

VwGO, 940 Rn. 278; Ruthig, DOV 1997, 399 If., \{41; 1. A. Eidermuller. EuZW 1995, 632 (f, 63\}.

s4 Seemer, Rechesbindungen und Rechisschutx bei der Vergabe offentlicher Aufurage, S. 127 I.

is BT-Drs. 12/4636, S. .

36 EuGHE 1997-1, 4961 , 4995 ; ebenso bercits KG, BauR 1995, 837, 842. 
völlig unabhängig sind, denn sogar ein nicht von einer staztlichen Srelle, sondern von den Parteien beserztes Schiedsgericht kann vorlageberechrigt sein, wenn es geserzlich vorgesehen ist. Auch die Frage, ob der Spruchkörper aus Berufsrichtern oder aus ehrenamtlichen Richtern bestehr, ist für den gemeinschafrsrechtlichen Gerichrsbegriff irrelevant. Lediglich eine offensichtliche Zuordnung zur mic der Sache vorbefaßten Exekutive wie im Fall der luxemburgischen Steuerverwaltung schließt einc Stelle von der Vorlageberechrigung aus. Der EuGH beschränke das Gebor der Unparteilichkeit also auf das Verbälenis zu staatlichen Stellen.

\section{Unparteilicbkeit als Konkretisierung des Prinzips der persönlichen Un- abbängigkeit}

Der Uberblick über die Rechtsprechung hat deuclich gemachc, daß das Merkmal der strukrurellen Unparteilichkeit zwar in verschiedenen Konsıellationen eine Rolle spielt, jedoch noch nichr präzise gefaßt werden konnce. Eine dogmatisch konsistente Definition der Anforderungen an die strukturelle Unparteilichkeit ist aber erforderlich, um dem Gerichtsverfassungsrechr eine klare Leiclinie zu geben. Zunächst wird die verfassungsreclitliche Basis des Gebotes struktureller Unparteilichkeit geprüft (t.). Anschließend werden die Schlußfolgerungen für das Gerichtsverfassungsrecht dargestellt (2.).

\section{Die Ableitung des Gebots der strukturellen Unparteilichkeit}

Die dargestel)te Rechisprechung der deurschen Gerichtc zeichnet sicl dadurch aus, daß der verlassungsrecheliche Prüfungsmaßstab außerordentlich vage bleibr. Neben Arr. 20 Abs. 2, 92 und 97 GG finden sich auch Entscheidunger, die Art. 19 Abs. 4 und 101 Abs. I S. 2 GG heranzichen. Meistens werden mehrere Bestimmungen kumulativ herangezogen. In anderen Fällen wird ohne konkrete Anknüpfung an eine Vorschrift der Verfassung betont, daß die Entscheidung durch einen neutralen Dritten zu den Wesensmerkmalen der Gerichtsbarkeir gehöres7. Hierin zeigen sich nicht nur Unklarheiten in der Sache. Auch die Schlußfolgerungen für den Spiclraum des Geseczgebers und für die Möglichkeit, die Frage der strukturellen Unparteilichkeit in einer Verfassungsbeschwerde geltend zu machen, hä̈gen von der verfassungsrechclichen Verankerung des Gebores ab.

Besonders weitreichende Konsequenzen hat es, wenn das Gewaltenteilungsprinzip des Art. 20 Abs. 2 GG herangezogen wird, da es durch die Unabänderlichkeitsgarantie in Art. 79 Abs. 3 GG einen besonders hohen Stellenwere hat. Dabei wird jedoch übersehen, daß das Grundgeseız auch im Bereich der Dritten Gewalt keine strikıe Funksionentrennung vorsieht ${ }^{88}$. Bei den ehrenamelichen Richtern besteht keineswegs eine ausnahmslose Unvereinbarkeir mic einem Amc in der Exekucive. Die öffentliche Verwaleung kann niche als einheitlicher Block behandelt werden, so daß ihre Be-

57 Vgl. Fn. 19; cbonso Acherberg (Fn. 12), An.92 Rn. 274; AK-GG-Wassermunn, 2. Aufl, An. 97 Rn. 13; Detuerbeck, in: Sachs (Hrsg.), GG, 2. Aufl, Art.92 Rn. 25; Heyde, HdbVerR, $\$ 33$ Rn. 7, 77 1.; Larenx. Der Rechesschutz des Bürgers und dic Rechesweggarantic, S. 22.

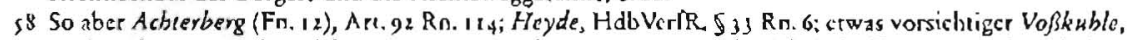
Rechisschutz gegen den Richter, S. 95 Fn. 36: iußerst geringe Durchlassigkeir der Trennungslinic z. wischen vollzichender und rechesprechender Gewalt. 
schäftigten in Streitigkeiten mit staatlicher Beteiligung stets als parteiisch anzusehen wären. Das Gewaltenteilungsprinzip ist im grundgesetzlichen System der Staatsorganisation nicht scarr vorgegeben, sondern ist in Randbercichen der Ausgestaltung durch einfaches Rechr zugänglich". Schlechterdings ausgeschlossen isc zwar, daß eine Sielle innerhalb der hierarchisch organisierten Venwaltung als Gericht anerkannt wird. Das Geboz struktureller Unparteilichkeit kann aber niche unmiutelbar aus Art. 20 Abs. 2 GG abgeleiret werden.

Die Begründung des Neucralitätsprinzips aus dem Wesen der Gerichtsbarkeit legt eine Anknüphung an den Gerichtsbegriff des Art. 22 GG nahe. Nach allgemeiner Auffassung ist neben der Stellung als besonderes Organ, das speziell zu dem Zweck geschaffen ist, Aufgaben staatlicher Rechtsprechung wahrzunehmen ${ }^{60}$, die sachliche Unabhängigkeit des Richters nach Art. 97 Abs. I GG ein notwendiges Merkmal der Gerichtsbarkeir ${ }^{62}$. Es ergibt sich unmittelbar aus der Verfassung, daß kein Richter Weisungea einer anderen Stelle unterworfen sein darf, abgesehen von bestimmten Fällen der Bindung an Entscheidungen anderer Gerichre ${ }^{6 *}$. Die sachliche Unabhängigkeit allein sichert die Neutralität der Gerichte jedoch nicht ausreichend, sie muß durch die persönliche Unabhängigkeit der einzelnen Richter ergänzt werden ${ }^{63}$.

Dieses Enfordernis ist allerdings niche ähnlich präzise zu fassen wie die sachliche Unabhängigkeit. Bescimmte Mindeststandards werden zwar für Berufsrichter durch Art. 97 Abs. 2 GG festgelegt, jedoch beschränken sie sich auf hauptamtlich und planmäßig endgülrig angestellre Richrer. Hieraus kann im Gegenschluß abgeleitet werden, daß andere Kacegorien von Richtern niche unzulässig sind. Zu ihnen zählen zum einen die Richter auf Probe, die Richter kraft Auftrags und die abgeordneten Richter, die auch als $*$ Hilfsrichter* bezeichnet werden ${ }^{64}$. Ebensowenig erfüllen zum anderen die ehrenamtlichen Richcer die Standards des A.r. 97 Abs. 2 GG, da sie nur eine begrenzte Amtszeit baben und nicht haupramtlich tätig sind. Dennoch werden auch Spruchkörper, die mehrheitlich oder im Ausnahmefall der Berufsgerichte der Anwälte ausschließlich mit Richtern besetzr sind, die keine auf Lebenszeit angestellten Berufsrichter sind, als Gericht im Sinne von Art. 92 GG anerkanne.

In diesen Fällen verwandelt sich die richterliche Unabhängigkeit von einer Rcgel mit eindeurigen Rechtsfolgen zu einem Prinzip, das der Ausfüllung durch den Geseczgeber bedarf's. Ihre Garantie kollidiert mit anderen Gesichtspunkten, die ebenfalls als legitim anerkannt werden. Für den Ausgleich beider Aspekte sind differenzierte Regelungen im Rahmen des richterlichen Statusrechtes und des Gerichtsverfassungsrechtes erforderlich. Für den Einsatz von Hilfsrichtern wird die Notwendigkeit eines flexiblen Personaleinsatzes und einer Nachwuchsauslese angeführt". Ihre Beteiligung an gerichtlichen Spruchkörpern ist deshalb zulässig, doch dürfen sie nur ausnahmsweise und nur aus zwingenden Gründen eingeseczt werden ${ }^{67}$. Dementsprechend wird ihr Einsarz durch \$\$28 f. DRiG eng begrenzt ${ }^{68}$. Für die ehrenamelichen Richter spricht das Prinzip der Laienbeteiligung als alter Forderung der demokrazi-




schen Bewegung des 19. Jahrhunderss ${ }^{69}$, die als Korrektiv lür die »déformation professionelle der Berufsjuristen auch heuce noch nicht obsolet is: ${ }^{70}$, und die Gewinnung von Sachverstand. Aus der Unabhängigkeirsgarantie ergibt sich jedoch, daß sie nach $\S_{44}$ Abs. 2 DRiG wie Berufsrichter vor Ablauf ihrer Amtszeit gegen ihren Willen nur durch eine gericlyliche Entscheidung abberufen werden können"'.

Damit ist auch der verfassungsrechtliche Standort des Gebotes strukrureller Unparteilichkeit gefunden ${ }^{7}$. Es reguliert den Bereich, in dem die personelle Unabliängigkeic dadurch relativiert ist, daß Richter zugelassen werden, die nicht allein aufgrund ihrer juriscischen Qualifikation für das Richzeramt ausgewähit wurden. Die Vorschriften über den Einsatz ehrenamtlicher Richter müssen folglich sicherstellen, daß Rollenkollisionen, die ihrc persönliche Unabhängigkeit gefährden, möglichst ausgeschlossen werden. Aus der Funktion der staatlichen Gerichtsbarkeit ergibc sich, daß die persönliche Unabhängigkeit der Richter nicht nur gegenüber anderen staatlichen Srellen gilt, sondern auch allgemein gegenüber den Parteien ${ }^{7 s}$. Die Unparteilichkeit der Richter muß im Sinne eines Distanzschutzes auch gegen urzulässige Einflüsse von Dritcen abgesichert werden". Das Vertrauen der Parceien in die gerichicliche Streitschlichtung ist davon abhängig, daß jede unsachliche, dic Neutralität beeinurächtigende Beeinflussung vermieden wird, unabhängig davon, ob sic von einer anderen staaclichen Stelle oder von gesellschaftlichen Kräfren ausgehc.

In diesem Sinne eines Prinzips, das niche absolut gilt, sondern relativierbar ist, ist das Gebot strukcureller Unparteilichkeit als generelle Anforderung an scaarliche Gerichue in Art. 92 GG verankert. Damir wird es zugleich durch die Garancie des geserzlichen Richters nach Art. 101 Abs. I S. 2 GG, die die Einhalcung der verfassungsrechrlichen Unabhängigkeitsanforderungen umfaßt, subjektivierc ${ }^{75}$. Geseczliche Regelungen, die die durch das Geboc der strukcurcllen Unparteilichkeit gezogenen Grenzen verlerzen, können ebenso wie die willkürliche Anwendung von Unvereinbarkeitsregeln, die das Gebot konkretisieren, durch Verfassungsbeschwerde gerügt werden.

\section{Die Anforderungen der strukturellen Unparteilichkeit}

Ausgangspunkt der Überlegungen, welche konkreten Anfordcrungen aus dem Geboc der strukturellen Unparteilichkeit abzuleiten sind, isı die Frage, in welchen Fällen eine besondere Nähe zu einer Partei vorliegh die die Stellung des Gerichts als neutraler Dritter in Frage stellt. Da es sich um allgemeine Probleme der Gerichiseigenschalt handelt, werden jeweils die europarechtichen Fragen mitbehandelt. Auch hierbei ist zwischen den beiden Fallgruppen der gruppen- und der funktionsbezogenen Auswahl zu unterscheiden.

\footnotetext{
$69 \mathrm{Vgl}$. Krbme, Dic Reichsverfassung der Paulskirche, 2. Aufl, S 359 ff.

to Vgl. die Diskussion bei Windel. ZZP 112 (1999), 293 ff., 297 ff., wobei sich die Frage sicllt, ob Bcrufsjuristen bei der Bcuncilung nicht strukturell pancilich sind.

71 BVCrfGE 14, 56, 70: 26, $186,1981, ; 87,68,85$

7. Den Zukammerhang rwischen Unabhangigkeir und Unparteilichkeit bceonen auch Frorvein/Peukere (Fn. 17), An.6Rn. 130, und Miehsler/Vogler (Fn. 18). Art. 6 Rn. 100; s. a. Rädler, in: WeissbroduNolfrum (Eds.), The Right to a Fair Trial, S. 727 if.

7) Eachenberger, Dic richterliche Unabhangigkcit als slaxisrechrliches Problem, S. 44; Siem (Fn. 13). S. gog; Janss/Pierath, GG, s. Auf., Art. 97 Rn.s

74 Schmidi-Aßmann (Fn, 22). Einlcitung Rn. 43.

7s V Vl. Degenlsare (Fn. S7), An. 101 Rn. $\$$ m, w. N.
} 
Das Problem der sirukturellen Unparteilichkeic von Richtern, die als Mitglieder bestimmter Gruppen ausgewählt werden, un in Prozessen, die die Interessen dieser Gruppen besonders becreffen, mitzuwirken, ist zwar wiederholc aufgeworfen worden, doch hat das Bundesverfassungsgeriche entsprechenden Bedenken bisher in keinem Fall staktgegeben. Auffällig ist dabei, daß die Entscheidungen keine subsrantielle Begründung für die Ablehnung einer unzulässigen Voreingenommenheir enthalten. Eine valide empirische Basis für die Beurteilung des Entscheidungsverhaltens ehrenamzlicher Richter gibr es bisher nicht ${ }^{76}$. Deshalb liegt die Vermutung nahe, daß das Verfassungsgericht den überkommenen Besetzungsvorschriften vor allem aufgrund ihrer langjährigen Tradition eine immanence Vernunfc zubilligt. Auch die von den Tarifparteien ausgewählten ehrenamelichen Richter in der Arbeitsgerichrsbarkeit werden in der Literatur aufgrund der gleichgewichtigen Beteiligung beider Interessengruppen und der persönlichen Neutralitätsverpflichrung überwiegend als unproblematisch angesehen" . Besonders großzügig ist der Gerichushof der Europäischen Gemeinschaften, der auch bestimmre Schiedsgerichte als vorlageberechtigt anerkannt hat.

Diese Entscheidungen zeigen, daß es kaum sachlich begründbare Maßstäbe für die Beurteilung der strukiurellen Unparteilichkeir gruppenbezogen ausgewählter Richter gibr. Lediglich eine offensichrlich unausgewogene oder sachwidrige Zusammenserzung eines Spruchkörpers kann ausgeschlossen werden. Innerhalb dieses weiten Rahmens ist eine politische Bewertung der einschlägigen Interessenstrukturen notwendig, die in der Verantwortung des Geseczgebers liegt ${ }^{78}$. Deshalb ist auch der im Fall Langborger angelegte Maßstab niche überzeugend. Offensichtlich war es die in anderen Ländern unbekannte Kollektivvertragsstruktur des schwedischen Mietrechtes, nicht aber die konkrece Besetzung des Gerichtes, die als problematisch angesehen wurde. Damir wird aber dor Beurceilungsspielraum des Mitgliedstaaces zu eng gezogen. Eine Anwendung der Grundsätze dieser Entscheidung auf andere Fälle scheidet aus.

\section{b) Schranken der Überschneidung exekutiver und judikativer Funktionen}

Die historische Scoßrichtung der Unabhängigkeitsgarantie isc in erster Linie auf die A bwehr von Ingerenzen durch andere staatliche Stellen bezogen 79 . Deshalb isc eine zu enge Verknüpfung einer Funktion in der Verwaltung mir dem Amt als ehrenamtlicher Richter in der zur Verwaltungskontrolle berulenen Gerichtsbarkeit ein Verstoß gegen das Gebot struktureller Unparteilichkeit und damit gegen das Prinzip der persönlichen Unabhängigkeit. Es muß sichergestellt werden, daß nicht Loyalitäten aus der administrativen Rolle die Unparteilichkeit der richterlichen Tärigkeit tangieren. Die

y6 Dic Ende der boer Jabre durchgefühne Umírage von KLassa (Fn. 6), S. 122 ff, fand kcine Bestàigung für cinc besondere Imeressenausrichtung der chrenamelichen Richter; îre Aussagckrafe ist jedoch durch dic Beschränkung auf Weseberlin und den Zcitablauf gering; skeptisch auch Stelkens (Fn. 66). \$ i Rn. 2 Fn. s. Eine empirische Untersuchung zu den Schöfen kam zu dem Ergebnis, daß es keine systcmatischen Unterschiede zu den Auffassungen der Benussricherer g(bo, vgl. Rennig (Fn. 7). S. 5886 .

77 Dazu ausfuhrlich Bengelsdorf, DB Bcilage $8 / 2987$ m.w.N.; vgl. wuch zur Sozialgcrichtsbarktit BLatzll Fromen, in: Entwicklung des Sozialrechts, Aufgabe der Rechesprechung. S. rop ff ; grundsütálich kritisch jext aber Windeh ZZP 112 (1999), 293 (f. 303 ff.

i8 Vgl. daru allgemein Groß (Fn.21). S. 2siff.

79 Riedel (Fח. 6), S. 221 fi, der allerdings dieses Argument bej der Auslegung von Art. 101 Abs. I S. 2 GG selbst relarivien (S. z41); s. a. Simon, Dic Unabhängigkeiz des Richters, S. I il?. 
geltenden geseczlichen Regelungen lassen jedoch ebensowenig wie die Rechtsprechung eine klare Linic erkennen, wie eng der sachliche Zusammenhang zwischen der exekuriven Funkcion und der richterlichen Zuständigkeir sein muß, um einen Verstoß gegen das Gebot der strukturellen Unparteilichkeit zu begründen. Der Gesezzgeber räumt insbesondere bei fachlich spezialisicrten Gerichten der Sachkunde der ehrenamtlichen Richter ein hohes Gewicht ein. Sie wird auch von der Rechtsprcchung regelmäßig als Rechtfertigung für die Beteiligung chrenamclicher Richter aus den Reihen der Verwaltung angeführr ${ }^{\circ \circ}$. Der Europäische Gerichrshof für Menschenrechce hat ebenfalls aus dem Gebor der Unparteilichkeit in Art. 6 Abs. I EMRK niche den Schiuß gezogen, daß alle exekuciven Funkcionsträger aus der Gerichrsbarkeit ausgeschlossen werden müssen. Auch der Gerichtshof der Europäischen Gemeinschaften sah beim Vergabeüberwachungsausschuß des Bundes die gleichzeitige Wahrnehmung von Verwaltungsaufgaben durch seine beamteren Mitglieder nicht als Hinderungsgrund für die Anerkennung der Gerichtsqualität an.

Das Erfordernis strukturelter Unparteilichkeit ist auch in diesem Kontext kein striktes Gebor, aus dem sich für alle Fälle ein eindeuriger Maßstab ableicen läßt. Zwischen der gewünschten Sachkunde und der nachteiligen Rollenkollision bedarf es viclmehr einer Abwägung, die nur durch den Geseczgeber erfolgen kann. In der allgemeinen Verwaltungsgerichrsbarkeit wird in einer sehr weitgehenden Regelung durch $\$ 22$ Nr. 3 VwGO allen Beamten und Angestellten des öffentichen Dienstes, soweit sie nicht ehrenamtlich tärig sind, die Berufung zum ehrenamtlichen Richter grundsäzzlich verwehr ${ }^{8}$. Zusärzlich erklärr $\$ s_{4} \mathrm{Abs} .3 \mathrm{VwGO}$ die Besorgnis der Befangenheit nach $\$ 4^{2}$ ZPO stets für begründet, wenn der Richter oder ehrenamtliche Richter der Vertretung einer Körperschaft angehört, deren Interessen durch das Verfahren berührt werden. Diese strikte Unvereinbarkeitsregelung geht eindeutig über das verfassungsrechtlich Geborene hinaus ${ }^{\delta_{2}}$. In der Finanzgerichtsbarkeit sind dagegen nach $\$ 19 \mathrm{Nr}, 3$ FGO nur Beamte und Angestelle der Sicuerverwaltungen des Bundes und der Länder von der Berufung zum ehrenamtlichen Richter ausgeschlossen. Die Inkompatibilitär beschränkt sich also auf den fachlich zuständigen Verwaltungszweig. Bci der Sozialgerichtsbarkeit wird in $\$ 17$ SGG noch stärker differenziert.

Das Gebor der strukturellen Unparteilichkeit enthält jedoch eine Mindestgarantie, die die verfassungsrechtlich notwendigen Inkomparibilitäten festlegt. Die Neutrálität des Gerichtes verlangt zwingend, daß Personen, die als Mitarbeicer einer Verwaltungsbehörde oder als Organmitglieder eincr Körperschaft, Anstalt oder Stiftung für besrimmte Angelegenheiren zustäodig sind, über sie nichr anschließend als ehrenamtliche Richter entsclseiden. Die Reichweice der Unvereinbarkeit orientient sich folglich an der Binnendifferenzierung der öffentlichen Verwaltung. Abgrenzungskricerium ist die Aufgabenzuordnung zu einer selbständigen Verwaltungseinheit durch die entsprechenden Zuständigkeitsvorschriften ${ }^{8_{3}}$, cin Kriterium, das über die konkrete Vorbefassung hinausgeht. Dadurch wird ausgeschlossen, daß eine für die vor Gericht zu entscheidende Angclegenheit zuständige Verwaltungseinheit in der dienstlichen Sphäre Einfluß auf den als ehrenamulichen Richter fungierenden Beamten nehmen kann.

80 BVerfGE 23, 83,91; 27,312.323; 22, 206, 210;54, 159, 166; BVcrwG Buchhol7 424.01 \$ 139 FlurbG Nr. 11, 12: NVDZ-RR 1991, 389 : BSGE 40, 130, 133 .

S, Zur Austegung val. Zickow (Fn. g), $\$: 2$ Rn. g fr. m.tw.N

82 Seckens (Fn. 66), \$22 Rn. \}: Ziekow (Fn. 9), \$22 Rn. 9 .

8) Ähnlich Zickowi, cbd. 
Ob eine generelle Inkompatibilität vorgesehen werden muß, die die Berufung eines Amtsträgers zum ehrenamtlichen Richter von vornherein ausschließr, oder ob ein Ausschlußgrund im Einzelfall ausreichend ist, hängt von der Zuständigkeit des jeweiligen Gerichts ab. Eine abstrakte Unvereinbarkeit ist nur erforderlich, wenn der administrative und der judikative Zuständigkeitsbereich übereinstimmen. Überschneiden sich dagegen belsördliche und gericheliche Zuständigkeic nur tejlweise, genügt cin Ausschluß in den Fällen, in denen das Gericht Angelegenheiten der Verwalıungseinheit zu entscheiden hat, in der der Richter ein exckutives Amt ausübc. Parallele Interessenlagen, etwa bei Urteilen mit finanziellen Auswirkungen für alle Gemeinden oder alle Krankenkassen, die über den Einzelfall hinaus von Bedeutung sind, reichen dagegen nicht aus, um eine unzulässige Parteilichkeic wegen einer Rollenkollision zu begründen ${ }^{\delta_{4}}$. Ein Organmitglied oder Beschäfrigter einer Körperschaft darf als Richter über Angelegenheiten eincr anderen Körperschaft entscheiden.

In der hierarchischen Sraatsverwaltung kommt es folglich auf die Behördengliederung an. Aufgrund der fehlenden Zuscändigkeissüberschneidung ist deshalb zutreffend die Mirwirkung von Beamten der Fachverwaltung in Flurbereinigungsgerichten anderer Bundesländer als zulässig angesehen worden ${ }^{85}$. Ebenfalls zutreffend ist nach diesem Maßstab - jedenfalls in bezug auf die Frage der Unparteilichkeir - die Anerkennung der Gerichtsqualitär des Bundesvergabcausschusses durch den Luxemburger Gerichushof, da das Bundeskarcellame selbst nicht für Vergaben zuständig ist. $\mathrm{Zu}$ weitgehend ist dagegen die Entscheidung des Bundessozialgerichtes, Beamte der Versorgungsverwaltung generell von der Mitgliedschaft in den entsprechenden Kammern der Sozialgerichisbarkeit auszuschließen ${ }^{86}$. Auch hier muß auf die konkrete Zuständigkeit des Beisiczers in einer Behörde, die mit der vor Gericht anhängigen Sache bcfaßt ist, abgestellt werden.

Da die Disziplinargerichte Fälle aus allen Teilen der Verwaltung eines Landes bzw. des Bundes behandeln, besteht eine unzulässige Nähebeziehung nur, wenn die Dienststelle des Beisizzers betroffen ist. Es ist folglich möglicl?, sich auf einen konkreten Ausschlußtatbestand für die Beamtenbeisitzer zu beschränken, wobci neben den Ausschlußgrund wegen einer personalrechelichen Zuständigkeit auch der Schutz der Neutralität des Beisitzers vor Pressionen im dienstlichen Bereich tritt. Deshalb ist es norwendig, die Unvereinbarkeit auf alle einer gemeinsamen hierarchischen Behördenspitze unterstellten Beamten zu esstrecken, so daß entgegen der Rechtsprechung ${ }^{\delta 7}$ ein Ministerium als einheidliche Dienststelle zu belıandeln isc.

\section{Komplementäre Schutzmechanismen der Unparteilichkeit}

Die vorangehende Untersuchung hat ergeben, daß das Gebor struktureller Unparteilichkeir aufgrund seines Prinzipiencharakters nur in engen Grenzen zwingende Unvereinbarkeitsregeln begründen kann, während in vielen Fällen der Sachnähe und Fachkunde von chrenamulichen Richrern ein höheres Gewichr eingeräume wird als ihrer Neutralität. Es verbleibr jedoch ein gewisses Unbchagen gegenüber der Bereiligung von Richtern mit einem verminderten Maß persönłicher Unabhängigkeit ${ }^{88}$. Die Objektivierungsfunktion der Judikative läßt eine pluralistische Besetzung als weniger

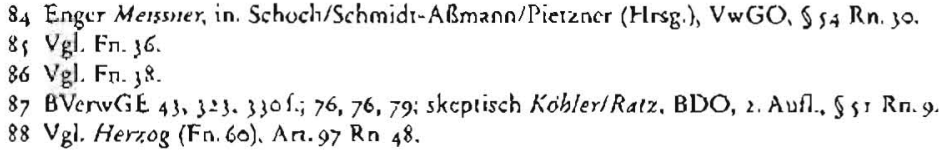


berechtigt als bei den Gestaltungsaufgaben der Exekutive erscheinen. Deshalb steltt sich die Frage, ab es andere Mechanismen gibt, um die Bedenken auszuräumen oder zumindest abzumildern. Die von der Rechtsprechung geforderte staatliche Auswahl der ehrenamtlichen Richter ( 1 .) und die Befangenheitsregeln (2.) erweisen sich hierfür jedoch als untauglich. Wirksam ist nur eine Kontrolle innerhalb der Judikative (3.).

\section{Die staatliche Auswabl der ebrenamtlichen Richter}

Als ein wesentlicher Mechanismus zur Sicherung der richcerlichen Neucralicäc wird die Personalauswahl angesehen. Das Bundesverfassungsgericht hat aus Art. 92 GG abgeieites, daß alle Gerichte im Sinne dieser Vorschrift staatliche Gerichre sein müssen. Dies erfordere nicht nur die Errichtung durch ein staatliches Gesetz, sondern auch, daß ihre personelle Besetzung entscheidend vom Staat bestimmt werden müs$s e^{89}$. Unter "Staat * werden dabei crsichtlich nur die Regierung auf Bundes- bzw. Landesebene und die ihr hicrarchisch nachgeordnecen Behörden verstanden, denn unter Berufung auf dieses Erfordernis wurde es als unzulässig angeschen, daß ein Teil der Mitglieder der Berufsgerichtsbarkeir einer beruf́sständischen Kammer mic Selbstverwaltungsrecht ohne Einschaltung von Regierungsstellen von der Vertreterversammlung der Kammer gewählt aurde ${ }^{\circ}$. Es sei zwar nicht notwendig, daß das Gericht organisatorisch der unmittelbaren Staatsverwaltung zugeordnet werde"'. Bei ihrer personellen Besetzung dürfe der Kammer jedoch allenfalls ein Vorschlagsrecht eingeräumt werden, das der staaclichen Justizverwalung ein auch quantitativ hinreichendes Auswahlrecht einräumen müsse ${ }^{92}$. Ebenso wurde für die Auswahl der ehrenamtlichen Richter in der A rbeits- und Sozialgerichtsbarkeit eine Bindung an dic Vorschläge der Verbände abgelehnt. Die staatliche Justizverwaltung müsse neben den gesetzlichen Vorausseczungen auch die Eignung der künfigen ehrenamelichen Richter prüfen können's.

Hiergegen wird allerdings eingewandr, daß es für eine solche Auswahlentscheidung kaum sachgerechte Kriterien geben könne ${ }^{94}$. Insbesondere darf selbstverständlich zum Schurz der richterlichen Unabhängigkeit nicht die rechtspolitische Linie der Vorgeschlagenen berücksichtigc werden. Auch Gesichtspunkte wic die soziale Ausgewogenheit's lassen sich kaum operationalisieren. Es gibr zudem keinen Grund zur Annahme, daß die Exckusive ein besonders gut geeigneter Wächter der gerichtlichen Neutralität ist ${ }^{96}$. Deshalb darf die Justizverwaltung nach zutreffender Ansicht nur die Einhaitung der gesetzlichen Vorausserzungen der Vorschlägc prüfen, aber keine weitergehende personenbezogene Auswahl vornehmen ${ }^{97}$.

Im übrigen ist die hierarchische Exekutive in anderco Fällen ganz aus dem Verfahren ausgeschlossen. Die Schöffen und die ehrenamelichen Richter in der Verwalcungsund der Finanzgerichrsbarkeic werden durch einen politisch legitimierten Ausschuß gewählc ( $\$ \$ 42,77$ GVG, $\$ 29$ VwGO, $\$ 26$ FGO), der eine Ermessensentscheidung

89 BVerfGE 18, 241, 293; BVeriG NJW 1986,1324 .

90 BV CriGE 18,241,254.

gr BVerIGE 14, 56,67; 18, 241, 253; a. A. Bctsermann, HSiR III, \$7? Kn. 73; v. Münch/Kunig-Meyer, GG, 3. Bd., 3. Aufl., Art. 92 Rn. 12.

22 BVeriGE 36, 186, ins li.; kriusch Larenr (Fr. 57), S. 222 ff,

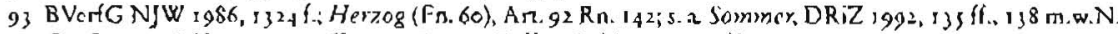

94 Großnann, SGb i985, 26; If, 267; Berger-Delhes; RdA 1988, 19 fi., 18,

9) Vgl. Kunt, ZZP 104 (1993), isolf., 162.

96 Groß, ZRP $1999,361 f_{-1} 301$ f.

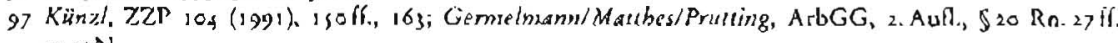
m.r.N. 
trifff, die nur bei sachfremden Erwägungen rechtswidrig, im übrigen aber niche kontrollierbar is ${ }^{98}$. Dieser Auswahlmechanismus anerkennt, daß cs keine verbindlichen Maßstäbe für dic Bestimmung der individuellen Eignung elrenamtlicher Richter gibc. Folglich kann aber auch ihr Auswahlmechanismus allein keine Gewälır für eine hinreichende Unparteilichkeit bieten.

\section{Die Befangenbeitsregeln}

Die Rechtsprechung verweist gelegentilich auf die Möglichkeit ciner Ablehnung des Richters im Einzelfall wegen Befangenheit ${ }^{99}$. Damit wird das Gebot der subjektiven Unparteilichkeit benannr, das sich auf den einzeinen Richter und einen konkreten Fall bezieht' ${ }^{10}$. Auch dieses Gebot wurzelt in der Unabhängigkeitsgarantie. Wie bei der strukturellen Unparteilichkeit kann aus der Verfassung jedoch lediglich das Gebor eines Mindestmaßes an Discanz gegenüber den Parceien abgeleiter werden, insbesondere der Ausschluß des Richters bei eigener Parteisrellung. Im übrigen bedarf das subjektive Neutralitätsgebot ebenfalls der geserzlichen Konkretisierung für die jeweiligen porentiellen Konfliktsituationen. Folgerichrig wird dieser Fragenkreis des individuellen Ausschlusses verfassungsrechelich im Schutzbereich der Garantie des gesetzlichen Richters angesiedelt ${ }^{\circ 0}$. Wie in den anderen Anwendungsfälien des Art. 10 r Abs. I S. 2 GG kommt es darauf an, daß dic normativ festgelegre Zuständigkeitsordnung nicht im Einzelfall aus sachfrenden Gründen manipuliert wird ${ }^{102}$.

Dieses Mittel greift in den Fällen einer generellen Rollenkollision jedoch nichı, da cine gesetzlich ausdrucklich zugelassene Distanzminderung für den Ausschluß nicht ausreichend sein kann, sondern ein individuelles, fallbezogenes Element hinzukommen muß ${ }^{103}$. Der Schurz der Zuständigkeitsordnung durch das Gebor des gesetzlichen Richters verlangt, daß eine struknurell bedingte Rollenkollision gerade nicht im Einzelfall, sondern durch geseizliche Inkompatibilitätsregelungen ausgeschlossen wird. Deshalb ist zu Recht abgelehnt worden, einen eltrenamclichen Arbeitsrichters wegen Befangenheir auszuschließen, weil er Funktionsträger in einer am Verfahren beteiligecn Gewerkschaft war, denn hierin manifestiert sich gerade das Interessengruppen-Prinzip ${ }^{104}$. Es gibe keine Zwischenlösung zwischen dem generellen Ausschluß einer gruppenbezogenen Auswahl von ehrenamclichen Beisirzern und dem carsächlich einzelfallbezogenen Ausschluß wegen Befangentzeir.

\section{Die Kontrolle durch böhere Gerichte}

Eine effekcive Kontrollmöglichkeit ergibr sich nur aus einem anderen Aspekt, der in der Rechrsprechung des Europäischen Gerichtshofes für Menschenrechte bereits angelegr ist. Er prüfre in den dargestellten Fällen das hier untersuchte Erfordernis der Unparceilichkeit jeweils nur in bezug auf das letzrinstanzlich entscheidende

\footnotetext{
98 Stelkens (Fn. 66), \$29 Rn. 2; Kopp/Schenke, VwGO, 1 . Aun., \$29 Rn. 2f; Ziekow (Fn 9), \$29 Rn. 1.7. gg $V_{g}$ l, 2. B. BVerfGE 23, 85, 21; 27, 311, 325; 42, 206, 211.

$100 \mathrm{Vgl}$. Wolf (Fn. 39), S. 221 if!



$102 \mathrm{Vgl}$. BVerfGF, 17, 294, 2y9.

10) So auch BAGE 20, 271, 27 \{; BSG SozR Nr.6 zu \$41 ZPO); Rieded (Fn. 6), S. 110,114 f.

104 BAG DB 1978,215 f
} 
Gericht. Besonders deutlich wurde dies im Fall Le Compte u.a., in dem das erstinstanzliche Berufsgericht eindeutig nicht den vom Gerichrshof aus Art. 6 Abs. 1 EMRK abgeleiteten Anforderungen entsprach, da es ausschließlich mit von den Ärzten aus ihrer Mitte gewählten Mitgliedern besetzt war. Der Straßburger Gerichtshof ließ es jedoch ausreichen, daß die zweite Inscanz ausreichend unparteilich war ${ }^{10 s}$. Auch das Bundesverfassungsgeriche hat angedeutec, daß bei erstinstanzlichen Gerichten ein großzügigerer Maßstab in bezug auf die Unparteilichkeit angelegt werden konnte ${ }^{\text {tcb }}$. Allerdings wurde diese Frage nie entscheidungserheblich.

Eine solche Überlegung widerspricht allerdings dem überwiegend vertretenen einheitlichen Gerichtsbegriff, der keine Differenzierungen zwischen verschiedenen Arten von Gerichten zulässt ${ }^{107}$. Es hat sich aber bereits gezeigt, daß die Verfassung im Bereich der Dritcen Gewalt bestimmen Unterscheidungen, z. B. zwischen Berufsrichtern und ehrenamtlichen Richtern, nicht grundsätzlich entgegensteht. Im Bereich des Gerichtsverfassungstechtes gibt es vielmelir eine große Vlelzahl von Ausgestaltungen der Spruchkörperbesetzung. Ein Verständnis der Gerichtsbarkeic als monolithischem Block überzeichnet die Rigidicät der grundgesetzlichen Regelung der Gewaltenteilung. Auch in der dritten Gewalt ist eine begrenzte Pluralisierung legitim. Auch die pauschale Formulierung des Art. $9_{2}$ GG, daß die rechesprechende Gewalt den Richtern anvertraut ist, meint offensichtich nicht, daß jedem Richter bedingungslos vertraut werden muß. Vielmehr kann auch die richterliche Gewalt niche von vornherein als kontrollfrei angesehen werden ${ }^{108}$. In den Fällen, in denen die immanenten Neutralitätsgarantien der Gerichtsbarkeit nur abgeschwächt greifen, weil Richter, die den Anforderungen des Art. 97 Abs. 2 GG nicht voll entsprechen, die Mehrheit des Spruchkörpers stellen, bedarf es der Möglichkeit einer Überprüfung durch ein höheres Gericht. Auch wenn einem generellen Mißtrauen gegenüber dem Einsatz ehrenamtlicher Richter ${ }^{109}$ nicht gefolgt werden kann, darf niche ignoriert werden, daß sie in höherem Maß neutralitätsgefährdenden Einflüssen ausgesetzt sind als Berufsrichter, zumal sic in der Regel nicht rechtskundig sind. Auch das Prinzip der Balance durch die antagoniscische Beseczung mit Vertetern von Interessengruppen kann versagen, zumal es niche durchgängig anwendbar ist, wie bei den landwirtschaftlichen Beisitzern in den Landwirtschafts- und Flurbereinigungsgerichren.

Deshalb ist es zum kompensatorischen Schutz der strukrurellen Unpartcilichkeit notwendig, daß in Fällen, in denen ausschließlich oder mebrheitlich mit ehrenamtlichen Richtern besetzte Spruchkörper entscheiden, zumindest die Revision an ein anderes Gericht möglich ist, das überwiegend mit Berufsrichtern beseczt ist. Eine solche Überprüfungsmöglichkeit bicter ein Gegengewichr zur Lockerung des Prinzips der persönlichen Unabhängigkeit und Neutralität, wenn sie im Interesse der Eunbeziehung gesellschaftlicher Kräfte vorgenommen wird. Diese Gestaltung des Rechtswegs garantiert einerseits, daß die besondere Fachkunde der ehrenamrlichen Richter in der Tassacheninstanz zur Geltung kommen kann. Sie mindert aber andererseits die Gefahr, daß dadurch rechtlich nicht zulässige Gesichtspunkte in die Entscheidung einfließen, da in jedem Fall eine Kontrolle der Rechtmäßigkeit der erstinstanzlichen Entscheidung erfolgen kann"'.

$\log \mathrm{V}_{\mathrm{g}} \mathrm{F}$. Fn. 22

106 BVerfGE 27, 312, \}2 s; \{2, 206, 110

107 Vgl. Stem (Fn. 13). S. 894: weinheirliche und geschlossene Gewnlte; ahnlich Riedel (Fn 6), S. 200.

108 Dazu ausführlich Voßkuble (Fn. s 8), S. 25 ff.; cbenso bereits in Bezug auf Veríahrensgamnticn Lorenz (Fr. 57), S. 2.41 $f$.

109 Vgl. z. B. Achuerberg (Fn. r2), Art. 92 Rn. 290 m.sv.N

110 Strenger Lippold. NJW 1991, 2583 if, 2389 , der im Fall des Einsaues von Probesichtern sogar eine zweite Tatsacheninsianz verlange. 
Die geltenden Prozeßordnungen entsprechen dicser Anforderung nichr durchgängig. Zwar ist in den meisten Fällen die Revisionsinstanz ausschließlich oder überwiegend mit Berufsrichtern besetzc (vgl. z. B. \$41 Abs. 2 ArbGG, $\$$ rob Abs. 2 S. I BRAO), in der Benfsgerichtsbarkeit ist dies aber nicht immer gewährleister (vgl.z. B. $\$ 21$ Abs. 2 S. I KammerG BW). In vielen Fällen sind zudem die Rechtsmittel durch bestimmte Zulassungsgründe begrenzt. Die rechtspolitische Tendenz, den Zugang zu höheren Instanzen aus Gründen der Beschleunigung von Gerichtsverfahren immer weiter einzuschränken'r', muß auch unter diesem Gesichtspunkt kritisch betrachtet werden.

Das Dogma, daß das Grundgesetz keinen Rechtsschutz gegen den Richter gewäh$\mathrm{re}^{\prime \prime 2}$, wird dadurch in einem Teilbereich zugunsten einer kompensatorischen Kontrolle innerhalb der Judikacive aufgegeben. Da aber Art. 97 Abs. 2 GG die auf Lebenszeit angestellten Berufsrichter besonders hervorhebc "1"3, darf das Prozeßrecht diese Entscheidung nicht dadurch konterkarieren, daß bestimme Fälle ausschließlich Spruchkörpern überlassen bleiben, in denen Berufsrichrer in der Minderheit sind. Die dritte Gewalt darf zwar in Einzelbereichen für gesellschaftliche Kräfte und für Angehörige der Exckurive geöffner werden, doch sind dann andere checks and balances notwendig, die das grundlegende Ziel des Rechtsstaates, die Bändigung staatlicher Macht, gewährleisten.

\section{Fazic}

Das Gebor scrukcureller Unparteilichkeir des Richters gilt nicht nur im Rahmen des Art. 6 Abs. I EMRK, sondern ist eine allgemeine Anforderung an die Gerichrsbarkeir, die auch im Rahmen des Arr. 92 GG und des Arc. 234 EG gilt. Es schließs eine offensichclich cinseitige gruppenbezogene Besetzung von Gerichten und die gleichzeitige Wahrnehmung von exekutiven und judikativen Funktionen im selben $\mathrm{Zu}$ ständigkeitsbereich aus. In anderen Fällen können Spruchkơrper jedoch auch mir Richtern beseczt werden, die nach speziellen Kriterien ausgewählt werden bzw. in der öffentlichen Verwaluung rätig sind, da das Prinzip der Ncutralicär der Judikative ciner Abwägung gegen die Ziele der Laienbereiligung und der Gewinnung von Sachverstand zugänglich ist. Als Sicherungsmechanismus isc es aber erforderlich, daß Spruchkörper, die überwiegend mit ehrenamtlichen Richtern beserzt sind, einer Kontrolle durch eine übergeordnete gerichuliche Instanz unterworfen sind, die mehrheitlich aus Berufsrichtem besetzt sind.

Das Urteil des Europäischen Gerichtshofs für Menschenrechte im Fall Langborger stellt folglich zu strenge Anforderungen an die strukturelie Unparteilichkeic. Insgesamt ist jedoch ein stärkeres Problembewußssein im Gerichesverfassungsrechı für die Grenzen der Pluralisierung der Judikative durcb ehrenamtliche Richter notwendig.

11 Vgl. zur 6. VwGO-Novelle Siner, DVBI. 1997, 326 If., 335 ff.; Meissner, VBIBW 1997, 81 ff.; Bader, NVWZ $1998,446 f f$.

112 BVeriGE 4.74. 96; $11,263.26$; Maunx/Dürig-Schmidt-Aßmann, GG, An. ig IV Rn. 96 m.w.N.i krit. auch Schurize-Fielize (Fn. 59), Bd. 1, An. 19 IV Rn. is.

11] Mcyer (Fn. 91), Arr. 91 Rn. 19: Normallalla. 